\title{
Power system parameters matching of in-wheel motor drive electric vehicle
}

\author{
Wei Chen Ren ${ }^{1, *}, Y_{i}$ Fang $^{1}$, Xiao Peng $\mathrm{Li}^{1}$, Zheng $\mathrm{Xu}^{1,2}$, Xu Long Wang ${ }^{1}$ \\ ${ }^{1}$ Tianjin New Energy Vehicle Driving Technology Engineering Center, Tianjin University of Technology and Education, Tianjin, \\ 300222, China \\ ${ }^{2}$ Donghexin Technology Co., Ltd., Tianjin 300350, China
}

\begin{abstract}
Based on the line-controlled chassis platform, the low-speed outer rotor motor is used to drive the wheel directly in the designed in-wheel motor line-controlled electric vehicle. The power system parameters of the designed four-wheel independent drive electric vehicle are matched and simulated by offline simulation. Firstly, based on the theoretical basis of the research on the parameter matching of the power system of pure electric vehicles, the type selection and parameter matching of the hub motor and power battery are carried out according to the established dynamic and economic indicators. Then, the designed component parameters are repeatedly corrected until the design goal is achieved; Finally, the vehicle model is built in AVL cruise, and the design results are simulated by software.
\end{abstract}

\section{Page layout}

With the continuous development of intelligent vehicle and intelligent transportation and smart city, the chassis technology of electric vehicles is changing in the direction of electric, intelligent, modular and lightweight. For the chassis bearing the motion performance of the vehicle, the wire control technology is used to carry out the overturning innovation, which greatly improves the dynamic performance and control performance of electric vehicles. On the basis of flexible connection of the linear control chassis, it is particularly suitable to use the in-wheel motor for the linear control drive of electric vehicles ${ }^{[1-2]}$. The wheel hub motor is a kind of drive form that installs the motor in the wheel, which simplifies the mechanical transmission device between the motor and the drive wheel, reduces the vehicle quality and the loss of energy transmission; it is able to drive, brake and steer four wheels independently and also can effectively avoid the resonance caused by the transmission mechanism under high speed response ${ }^{[3]}$, thus pave the way for the modular development of electric vehicles.

For improving the dynamic and economic performance of pure electric vehicles, the reasonable matching and selection of parameters play a very important role ${ }^{[4]}$. In this paper, the hub motor and power battery of the vehicle are selected according to the requirements and design objectives. After repeated design, the software simulation is carried out in AVL cruise.

\section{Parameter matching of power system for in-wheel motor drive electric vehicle}

\subsection{Vehicle Parameters and Performance Requirements}

The electric vehicle studied in this paper benchmarks the line-controlled chassis model produced by a domestic company, and sets the dynamic performance and economic performance according to the vehicle use and the positioning of the model. In general, the reasonable range of the maximum speed of micro electric vehicles should be $80-100 \mathrm{~km} / \mathrm{h}$, the design objective of this paper is $\geq 80 \mathrm{~km} / \mathrm{h}^{[5]}$. The reasonable range of the endurance mileage should be 100-120 $\mathrm{km}^{[5]}$, the design objective of this paper is $\geq 100 \mathrm{~km}$. And the maximum climbing degree of ordinary cars should be about $30 \%$. The acceleration ability of the designed electric vehicle on the horizontal road is referred to the Euler R1 model.

The full load mass of the electric vehicle studied in this paper is $350 \mathrm{~kg}$; the transmission efficiency of the hub motor is 0.95 ; the rolling resistance coefficient is 0.015 ; the air resistance coefficient is 0.4 ; the windward area is $1.4 \mathrm{~m}^{2}$; the rotation mass conversion coefficient is 1.03 ; tire model is $145 / 70 \mathrm{R} 12$.

*Corresponding author: 1095018245@qq.com 


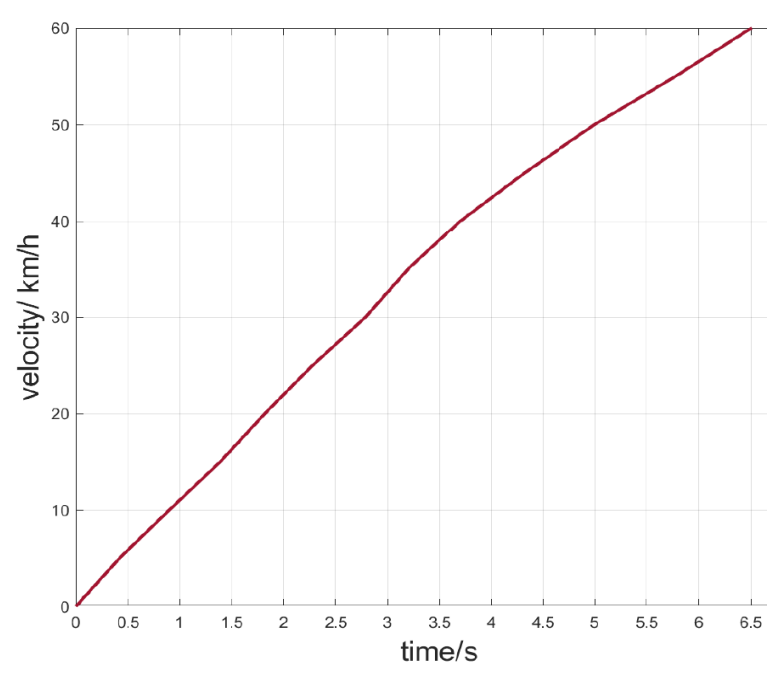

Fig. 1. 0-60 km/h acceleration time of Euler R1

\subsection{Selection and Parameter Matching of Hub Motor}

Permanent magnet synchronous motor (PMSM) is an ideal driving motor with high power factor, high efficiency and high power density. Therefore, this paper selects PMSM as the source of driving force. According to the established dynamic and economic indicators, the power, torque and speed of the motor are designed.

\subsubsection{Power of hub motor}

When the vehicle is fully loaded and runs at the highest speed, the total output power of the hub motor is:

$$
P_{1}=\frac{1}{\eta}\left(\frac{m g f}{3600} u_{\max }+\frac{C_{D} A}{76140} u_{\max }{ }^{3}\right)
$$

$g$ is the gravity acceleration; $u_{\max }$ is the maximum speed of the vehicle. According to formula (1), the total output power of the hub motor is about $5.2 \mathrm{~kW}$.

When the vehicle is fully loaded, the power consumed by climbing $30 \%$ of the slope at a speed of $20 \mathrm{~km} / \mathrm{h}$ is:

$$
P_{2}=\frac{1}{\eta}\left(\frac{m g f}{3600} \cos \alpha_{\max } u_{a}+\frac{m g}{3600} \sin \alpha_{\max } u_{a}+\frac{C_{D} A}{76140} u_{a}^{3}\right)
$$

$\alpha_{\max }=\arctan (0.3)$ is the maximum climbing angle, $u_{a}$ is $20 \mathrm{~km} / \mathrm{h}$. The in-wheel motor power calculated by formula (2) is about $6.3 \mathrm{~kW}$.

From static acceleration to $40 \mathrm{~km} / \mathrm{h}$ and the acceleration time is controlled at $4 \mathrm{~s}$, the total power is:

$$
P_{3}=\frac{1}{\eta}\left\{\frac{m g f}{3600} u_{m 1}+\frac{C_{D} A}{76140} u_{m 1}{ }^{3}+\frac{\delta m u_{m 1} 1^{2}}{3600 d t}\left[1-\left(\frac{t_{m 1}-d t}{t_{m 1}}\right)^{x}\right]\right\}
$$

When the vehicle is fully loaded, from static acceleration to $60 \mathrm{~km} / \mathrm{h}$ and the acceleration time is controlled at $7 \mathrm{~s}$, the total power is:

$$
P_{4}=\frac{1}{\eta}\left\{\frac{m g f}{3600} u_{m 2}+\frac{C_{D} A}{76140} u_{m 2}{ }^{3}+\frac{\delta m u_{m 2}{ }^{2}}{3600 d t}\left[1-\left(\frac{{ }^{t} 2_{2}-d t}{t_{m 2}}\right)^{x}\right]\right\}
$$

In the formula, $u_{m 1}$ is $40 \mathrm{~km} / \mathrm{h}, u_{m 2}$ is $60 \mathrm{~km} / \mathrm{h}$, $t_{m 1}$ is $4 \mathrm{~s}, t_{m 2}$ is $7 \mathrm{~s}, d t$ is iterative step length, it is 0.1. $x$ is fitting coefficient, 0.2. According to the formula (3) (4), the total power of the hub motor is about $9.6 \mathrm{~kW} 、 12 \mathrm{~kW}$.

In general, the maximum total power output of the in-wheel motor should be greater than the consumed power under the above four conditions, $P_{\max } \geq \max \left\{P_{1}, P_{2}, P_{3}, P_{4}\right\}$. The maximum power of the drive motor is usually determined according to the acceleration performance of the electric vehicle ${ }^{[6]}$, $P_{\max }=12 \mathrm{~kW}$. The overload coefficient of the selected motor $\lambda$ is 2 , the rated total power of the hub motor is $P_{e}=P_{\max } / \lambda=6 \mathrm{~kW}$, the rated power meets the power requirements of the maximum speed of the vehicle ${ }^{[7]}$.

\subsubsection{Speed of hub motor}

The maximum speed of the in-wheel motor is determined by the maximum speed of the electric vehicle, and it is also the maximum speed in the constant power region in the characteristic curve of the driving motor ${ }^{[8]}: \quad n_{\max }=u_{\max } \frac{i}{0.377 r}$

$n_{\max }$ is the highest speed of the hub motor, the transmission ratio $i$ is set to 1 , and $n_{\max }$ can be obtained as $840 \mathrm{r} / \mathrm{min}$. The expansion of constant power coefficient $\beta$ is generally the most appropriate between 2 and $4, \beta=2$, the rated speed of hub motor is $n_{e}=\frac{n_{\max }}{\beta}=420 \mathrm{r} / \mathrm{min}$, and the vehicle speed is $40 \mathrm{~km} / \mathrm{h}$.

\subsubsection{Torque of hub motor}

To meet the starting and maximum climbing requirements of electric vehicles driven by in-wheel motors, the total peak torque calculation formula of inwheel motors is:

$$
T_{\max }=\frac{r\left(m g f \cos \alpha_{\max }+m g \sin \alpha_{\max }+\frac{C_{D} A}{21.15} u_{a}^{2}\right)}{i \eta}
$$

The total rated torque of hub motor is generally calculated by rated total power and rated speed:

$$
T_{e}=9550 \frac{P_{e}}{n_{e}}
$$

After calculation by Formulas (6)and (7), the total peak torque of the motor is $284 \mathrm{~N} \cdot \mathrm{m}$, and the total rated torque is $136 \mathrm{~N} \cdot \mathrm{m}$. The parameters of the four hub motors are distributed according to the distribution of the front and rear loads of the vehicle at 50:50.

\subsection{Power battery selection and parameter matching}

In this paper, the power battery type of the in-wheel motor drive electric vehicle is ternary lithium battery. The total voltage, total capacity and total energy of the battery pack should be considered when selecting the power battery, and the relevant parameters of the single battery should be combined. 
The voltage of the battery pack should be matched with the voltage of the drive motor. Within the input voltage range of the drive motor, the efficiency of the drive motor system increases first and then decreases with the increase of the voltage of the power battery pack $^{[9]}$. The rated voltage of the power battery pack is $U_{\text {ess }}=72 \mathrm{~V}$.

With the increase of the total capacity of the power battery, the endurance mileage of the vehicle is on the rise, but the increase of the battery quality is accompanied by the increase of the vehicle quality, unit mileage power consumption and cost. The gain brought by the increase of the power battery capacity is offset by the loss brought by the increase of the vehicle quality ${ }^{[5]}$. Therefore, this paper only considers the total energy and capacity of the battery pack to meet the design requirements of the vehicle at the speed of $40 \mathrm{~km} / \mathrm{h}$ for $100 \mathrm{~km}$, the total energy of the battery pack $W_{\text {ess }}$ should meet:

$W_{e s s} \geq \frac{P_{40} S}{D O D \eta_{b} \eta_{m c} u_{40}}=\frac{1}{D O D \eta_{b} \eta_{m c} \eta}\left(\frac{m g f}{3600}+\frac{C_{D} A}{76140} u_{40}{ }^{2}\right) S$

In the formula, the power at constant speed $P_{40}$ is $40 \mathrm{~km} / \mathrm{h}$, the endurance mileage requirement at current speed $S$ is $100 \mathrm{~km}$, the discharge depth of battery pack $D O D$ is 0.94 ,the discharge efficiency of battery pack $\eta_{b}$ is 0.92 , the electronic control efficiency of $\eta_{m c}$ is 0.96, $u_{40}$ is $40 \mathrm{~km} / \mathrm{h}$. According to Formula (8), the total energy of the battery pack calculated is $W_{\text {ess }} \geq 3.6 \mathrm{kWh}$

According to the calculated total energy requirements of the battery pack, the total capacity requirements of the battery pack is:

$$
C_{\text {ess }} \geq \frac{1000 W_{\text {ess }}}{U_{\text {ess }}}
$$

According to formula (9), the total capacity requirement of the battery pack is $C_{\text {ess }} \geq 50 \mathrm{Ah}$.

In this paper, a single cell with a nominal voltage of $3.6 \mathrm{~V}$ and a single cell capacity of $5 \mathrm{Ah}$ is used. Ten cores are paralleled to form a core group with a capacity of $50 \mathrm{Ah}$. Then 20 core groups are connected in series to form a battery pack with a voltage of $72 \mathrm{~V}$ and a total energy of $3.6 \mathrm{kWh}$. The battery pack can meet the design requirements of total energy and total capacity.

\section{Vehicle model establishment and simulation analysis based on AVL cruise}

\subsection{Establishment of vehicle model based on AVL cruise}

AVL cruise software can quickly build the complex power transmission system model, and conduct forward or reverse simulation analysis on the power and economy of the vehicle ${ }^{[10-11]}$. Based on AVL cruise, this paper builds the model of the designed in-wheel motordriven electric vehicle.

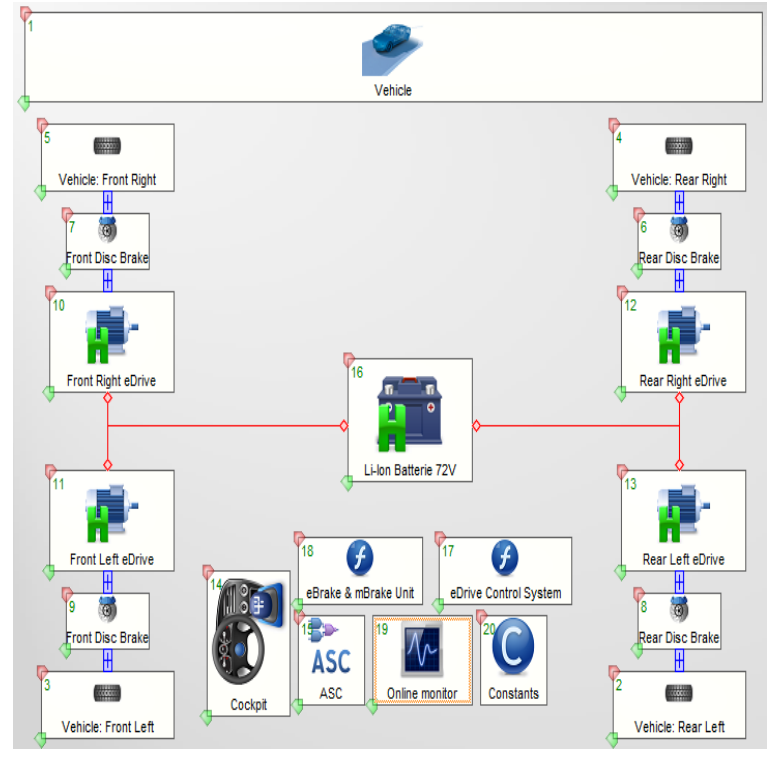

Fig. 2. Vehicle model based on AVL cruise

\subsection{Analysis of simulation results}

\subsubsection{Dynamic simulation results analysis}

The maximum speed simulation results of the in-wheel motor drive electric vehicle designed in this paper are $82.20 \mathrm{~km} / \mathrm{h}$. The maximum speed of the vehicle is mainly limited by the peak speed of the driving motor. When the vehicle reaches the maximum speed, the speed is $838.62 \mathrm{r} / \mathrm{min}$. The error between the simulation results and the designed maximum speed of $80 \mathrm{~km} / \mathrm{h}$ is less than $5 \%$, and the simulation results are reliable.

From the simulation results of acceleration time in Figure 4 , it can be seen that the acceleration time of $0 \sim$ $40 \mathrm{~km} / \mathrm{h}$ is $3.89 \mathrm{~s}$, and the acceleration time of $0 \sim 60$ $\mathrm{km} / \mathrm{h}$ is $6.58 \mathrm{~s}$. The simulation results are basically consistent with Euler R1, which meets the design goals.

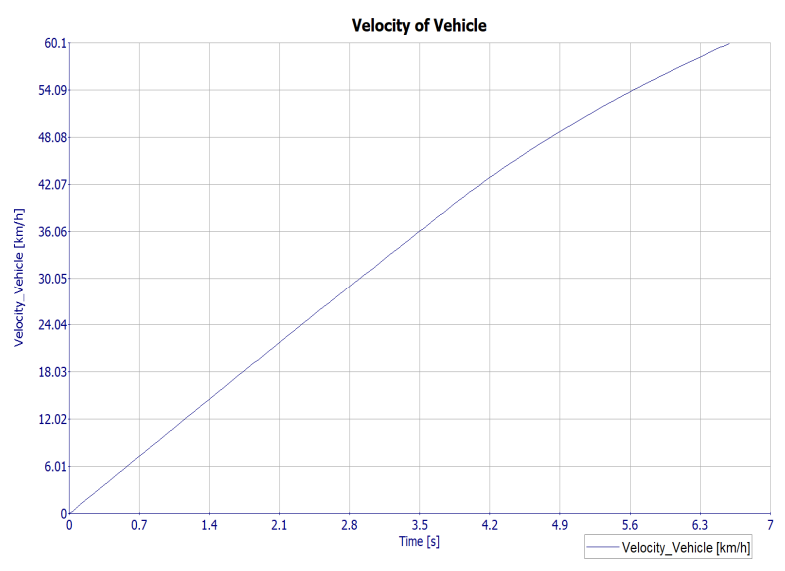

Fig. 3. Simulation calculation of acceleration time

From the simulation results of maximum climbing in Figure 5, when the vehicle speed is $20 \mathrm{~km} / \mathrm{h}$, the maximum climbing degree is $31.56 \%$, which is greater than $30 \%$ of the design, which meets the design goal. 


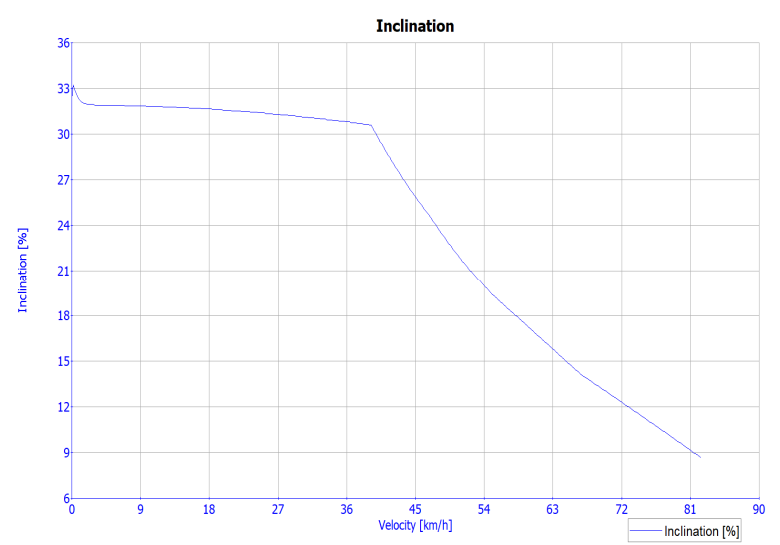

Fig. 4. Simulation of maximum climbing

\subsubsection{Economic simulation results analysis}

On the basis of the NEDC condition, the simulation results of the vehicle under the modified NEDC 1 condition are shown in Figure 6 and Figure 7. Within the allowable speed range, the required speed is basically consistent with the actual speed.

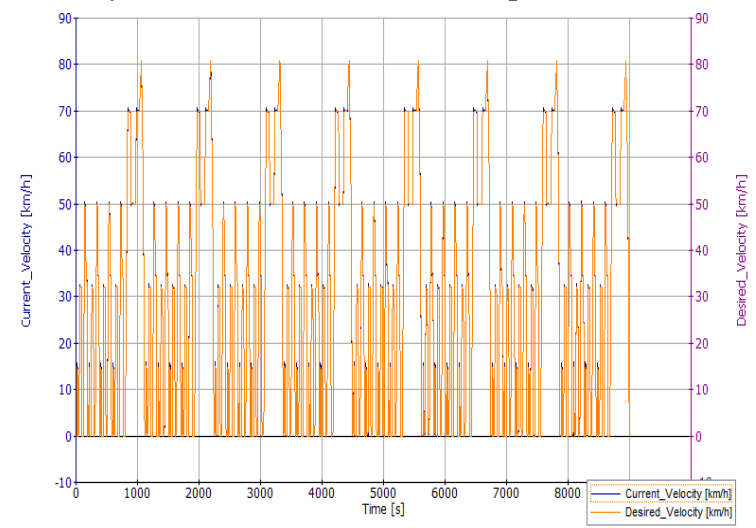

Fig. 5. Demand speed and actual speed

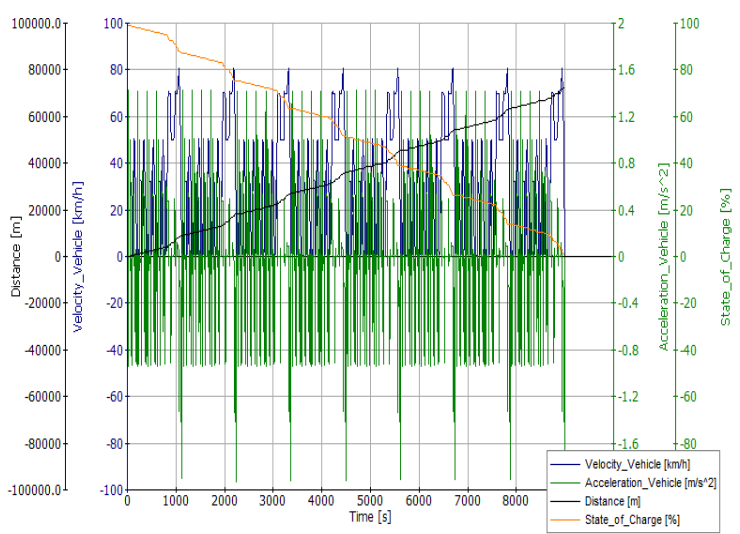

Fig. 6. The endurance mileage, vehicle speed and acceleration

When the designed vehicle runs at a constant speed of $40 \mathrm{~km} / \mathrm{h}$, the energy consumption rate is 32.8 $\mathrm{Wh} / \mathrm{km}$ and the endurance mileage is $111 \mathrm{~km}$, as shown in figure 8. Although the eDrive model in AVL cruise takes into account the second quadrant characteristics of the driving motor, based on the constant speed driving in the simulation condition, the reason that the data of simulation results considering the endurance mileage is larger may be that the vehicle is stable at low speed or the input voltage of the power battery pack makes the drive motor system in a high efficiency area. It may also be caused by the error of the power battery discharge depth, discharge efficiency and other data in the data matching process and the failure to consider the optimal working range of $\mathrm{SOC}^{[9-11]}$.

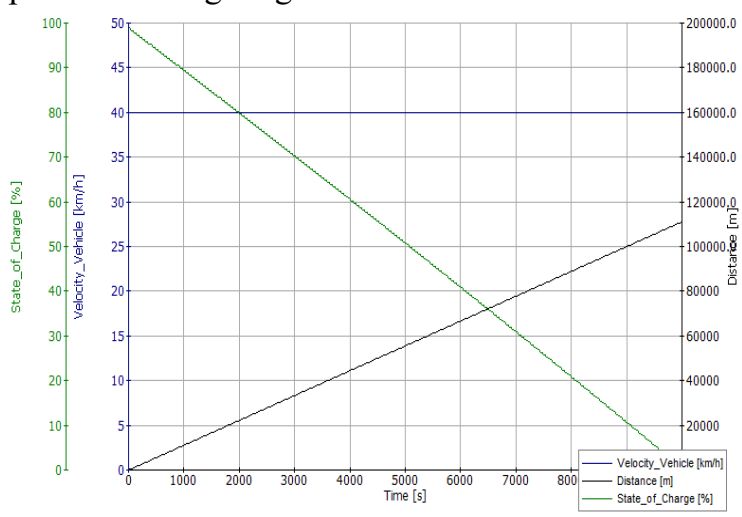

Fig. 7. Simulation of endurance mileage at constant speed

\section{4 conclusion}

- According to the established dynamic and economic indicators, the permanent magnet synchronous motor and the lithium battery were selected and the relevant parameters were matched, and the designed component parameters were repeatedly corrected;

- The in-wheel motor drive vehicle model is built in AVL cruise, and the design results are simulated;

- The results of simulation experiments verify the designed dynamic and economic indicators, and prove the correctness of the matching of motor and battery parameters.

\section{References}

1. C. Yang, C. Yan, Auto.Abs,12,13-19 (2019)

2. Z.C.F, L. Gang, Z.H.Y, H.L, Z.Z.X, Chi. Jou. Of. Hig, 26,2,160-176 (2013)

3. T.T.E.D, Electric Vehicles (Volume 10), S.P, (2020)

4. H.J.J, G. chen, Q.D.T, T.J. J. O. C. Q. U, 4,365-370 (2008)

5. G.J, O.M.G, L.L.G, L.J.Q, Chi. Jou. Of. Hig, 1, 177-183 (2013)

6. C.L, J.Z, 2011 ICICE, IEEE (2011)

7. C.X, J.J.N, F.P, $20112^{\text {nd }}$ AIMSEC, USA: IEEE, 8$10(2010)$

8. L.Y.L, Zhong Bei University (2020)

9. H.W.Y, C. Yang, C. Hong, W.H.D, J.O.J.S.U ( N.S.E ), 34,2,131-137 (2013)

10. Y.F.L, Z.J.H, Z.N.H, Z.G.Z, Auto. Eng, 36, 6, 669$672(2014)$

11. Y.Y.N, Z.Z.Y, Z.T, Z.X.Y, Auto.Eng,10,3943(2019) 